\title{
Discrete self-similar multifractals with examples from algebraic number theory
}

\author{
by \\ L. Olsen (St. Andrews)
}

1. Introduction and statement of results. A self-similar set is a set that can be decomposed into a union of subsets which are scaled down copies of the whole set. Similarly, a self-similar measure is a measure that can be decomposed into a sum of measures which are scaled down copies of the original measure. Self-similar sets such as the classical Cantor set, the von Koch curve and the Sierpiński triangle are amongst the most well known examples of fractal sets. Similarly, self-similar measures such as the binomial Cantor measure (cf. Figure 1 below) are amongst the most well known examples of fractal measures. Such fractal measures are called multifractal measures (or multifractals) and the study of the fractal properties, and in particular, the study of the so-called $L^{q}$-multifractal spectra, of these measures is referred to as multifractal analysis.

The basic ideas leading to the study of multifractal measures and their $L^{q}$-multifractal spectra originate from the work of theoretical physicists [HJKPS] in the 1980's, and during the past 20 years multifractal measures and, in particular, self-similar multifractal measures in $\mathbb{R}^{d}$ and their fractal structure have attracted an enormous interest in the mathematical literature. Indeed, the $L^{q}$-multifractal spectra of self-similar measures in $\mathbb{R}^{d}$ are by now well understood (cf. for example [AP, St] or the textbooks [Ed, Fa] and the references therein).

In this paper we will attempt to define self-similar multifractal measures in a discrete, and typically algebraic, setting and study their fractal structure. Firstly, we will provide definitions of a self-similar measure and its $L^{q}$-multifractal spectrum in a discrete (algebraic) setting; cf. Section 1.2 and, in particular, (1.5)-(1.7). Secondly, in Theorem 1.1 we obtain a formula for the $L^{q}$-multifractal spectrum of a self-similar multifractal in a discrete

2000 Mathematics Subject Classification: 11R04, 11K99, 28A80.

Key words and phrases: fractals, multifractals, self-similar fractals, self-similar multifractals, $L^{q}$-multifractal spectrum, algebraic numbers, ideals. 
(algebraic) setting. This formula is analogous to the classical formula for the $L^{q}$-multifractal spectrum of an ordinary self-similar measure in $\mathbb{R}^{d}$. Thirdly, we provide a number of illustrative examples taken from algebraic number theory: in Section 2 we consider self-similar fractal measures of ideals of number fields, and in Section 3 we consider self-similar fractal measures on polynomial rings over finite fields.

1.1. Self-similar measures in $\mathbb{R}^{d}$. To motivate our definitions and results, we begin by a brief description of self-similar measures in Euclidean space and their $L^{q}$-multifractal spectra. Therefore, let $d$ be a positive integer. A map $S: \mathbb{R}^{d} \rightarrow \mathbb{R}^{d}$ is called a similarity if there exists a positive real number $r>0$ such that

$$
|S(x)-S(y)|=r|x-y|
$$

for all $x, y \in \mathbb{R}^{d}$. The number $r$ is called the contracting ratio of $S$. If $r<1$, the map $S$ is said to be contractive. In 1981 Hutchinson [Hu] proved that finite families of contracting similarities can be used for generating self-similar measures.

Definition $([\mathrm{Hu}])$. Let $\left(S_{1}, \ldots, S_{m}\right)$ be a finite family of contracting similarities in $\mathbb{R}^{d}$ and let $\left(p_{1}, \ldots, p_{m}\right)$ be a probability vector. A Borel probability measure in $\mathbb{R}^{d}$ is called self-similar with respect to the family $\left(S_{i}, p_{i}\right)_{i}$ if

$$
\mu=\sum_{i} p_{i} \mu \circ S_{i}^{-1}
$$

For each finite family $\left(S_{1}, \ldots, S_{m}\right)$ of contracting similarities in $\mathbb{R}^{d}$ and each probability vector $\left(p_{1}, \ldots, p_{m}\right)$, there exists a unique Borel probability measure $\mu$ satisfying (1.1).

During the 1980's and 1990's self-similar measures, and generalizations thereof, have attracted a lot of interest. In particular, there has been an enormous amount of literature investigating the $L^{q}$-multifractal spectra of these measures. The $L^{q}$-multifractal spectrum of a measure $\mu$ is defined as follows. For a real number $q$ and $r>0$, write

$$
I_{\mathrm{R}}^{q}(r)=\int \mu(B(x, r))^{q-1} d \mu(x) .
$$

We now define the lower and upper $L^{q}$-multifractal spectra, sometimes also called the lower and upper Rényi spectra, of $\mu$ by

$$
\begin{aligned}
& \underline{\tau}_{\mathrm{R}}(q)=\liminf _{r \searrow 0} \frac{\log I^{q}(r)}{-\log r}=\liminf _{r \searrow 0} \frac{\log \int \mu(B(x, r))^{q-1} d \mu(x)}{-\log r}, \\
& \bar{\tau}_{\mathrm{R}}(q)=\limsup _{r \searrow 0} \frac{\log I^{q}(r)}{-\log r}=\limsup _{r \searrow 0} \frac{\log \int \mu(B(x, r))^{q-1} d \mu(x)}{-\log r} .
\end{aligned}
$$


The main result is the following theorem providing an explicit formula for $L^{q}$-multifractal spectra of a self-similar measure. This result appears explicitly in [AP, St] but is also implicit in [Ra]. For a more thorough discussion of this result and a more comprehensive list of references the reader is referred to $[\mathrm{Fa}, \mathrm{Lau}]$.

Theorem A ([AP, St, Ra]). Let $\left(S_{1}, \ldots, S_{m}\right)$ be a finite family of contracting similarities in $\mathbb{R}^{d}$ and let $\left(p_{1}, \ldots, p_{m}\right)$ be a probability vector. Let $r_{i}$ denote the contracting ratio of $S_{i}$. Let $\mu$ be the unique self-similar measure associated with the family $\left(S_{i}, p_{i}\right)_{i}$ and write $K$ for the support of $\mu$. Assume that the following condition is satisfied.

(i) $S_{i}(K) \cap S_{j}(K)=\emptyset$ for all $i \neq j$.

For each $q \in \mathbb{R}$, define the real number $\beta(q)$ by

$$
\sum_{i} p_{i}^{q} r_{i}^{\beta(q)}=1
$$

Then

$$
\underline{\tau}_{\mathrm{R}}(q)=\bar{\tau}_{\mathrm{R}}(q)=\beta(q) .
$$

Example. The classical ternary Cantor $C$ is defined by

$$
C=\left\{\sum_{l=1}^{\infty} \frac{a_{l}}{3^{l}} \mid a_{1}, a_{2}, \ldots=0,2\right\} .
$$

Let $\left(p_{0}, p_{2}\right)$ be a probability vector. It follows by a standard argument that there exists a unique probability measure $\mu$ on $C$ such that

$$
\begin{array}{r}
\mu\left(\left\{\frac{a_{1}}{3}+\frac{a_{2}}{3^{2}}+\cdots+\frac{a_{n}}{3^{n}}+\sum_{l=n+1}^{\infty} \frac{d_{l}}{3^{l}} \mid d_{n+1}, d_{n+2}, \ldots=0,2\right\}\right) \\
=p_{a_{1}} \cdots p_{a_{n}}
\end{array}
$$

for all positive integers $n$ and all $a_{1}, \ldots, a_{n}=0,2$. The measure $\mu$ is known as the binomial Cantor measure and is sketched in Figure 1 for $\left(p_{0}, p_{2}\right)=$ $\left(\frac{2}{3}, \frac{1}{3}\right)$. The measure $\mu$ is the standard example of a self-similar measure. Indeed, if we define $S_{0}, S_{2}: \mathbb{R} \rightarrow \mathbb{R}$ by $S_{0}(x)=\frac{1}{3} x$ and $S_{2}(x)=\frac{1}{3} x+\frac{2}{3}$, then clearly $\mu=p_{0} \mu \circ S_{0}^{-1}+p_{2} \mu \circ S_{2}^{-1}$. Since also $S_{0}(C) \cap S_{2}(C)=\emptyset$, it follows from Theorem A that $\underline{\tau}_{\mathrm{R}}(q)=\bar{\tau}_{\mathrm{R}}(q)=\beta(q)$, where $\beta(q)$ is the solution to the equation $\sum_{i=0,2} p_{i}^{q}\left(\frac{1}{3}\right)^{\beta(q)}=1$, whence

$$
\underline{\tau}_{\mathrm{R}}(q)=\bar{\tau}_{\mathrm{R}}(q)=\frac{\log \left(p_{0}^{q}+p_{2}^{q}\right)}{\log 3} .
$$

1.2. Self-similar measures on normed structures. The purpose of this paper is to provide an analogous definition of a self-similar measure $\mu$ in various discrete algebraic settings and to obtain a formula for the $L^{q}$-multifractal 

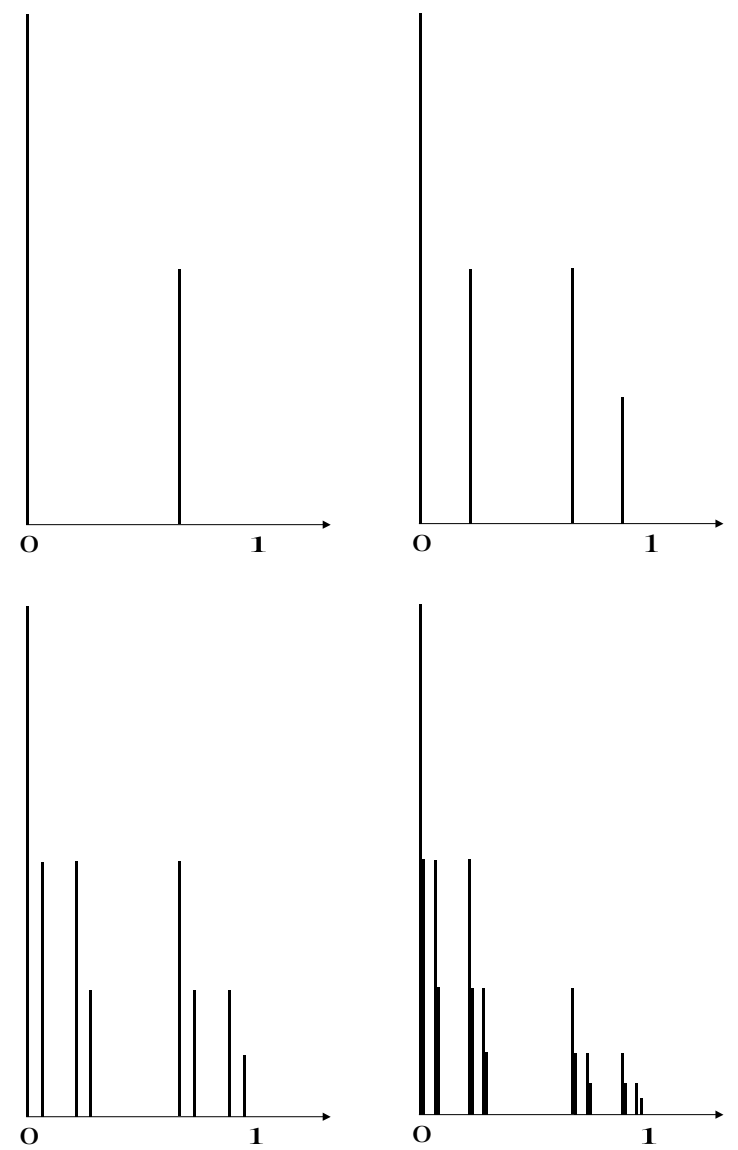

Fig. 1. This figure shows the first four measures $\mu_{1}, \ldots, \mu_{4}$ in a sequence $\left(\mu_{n}\right)_{n}$ of probability measures converging weakly to the binomial Cantor measure. The measures $\mu_{n}$ are defined as follows. Define $S_{0}, S_{2}: \mathbb{R} \rightarrow \mathbb{R}$ by $S_{0}(x)=\frac{1}{3} x$ and $S_{2}(x)=\frac{1}{3} x+\frac{2}{3}$, and put $\left(p_{0}, p_{2}\right)=\left(\frac{2}{3}, \frac{1}{3}\right)$. Then $\mu_{n}=\sum_{i_{1}, \ldots, i_{n}=0,2} p_{i_{1}} \cdots p_{i_{n}} \delta_{S_{i_{1}} \cdots S_{i_{n}}(0)}$ where $\delta_{x}$ denotes the Dirac measure concentrated at $x$. The height of each vertical bar is proportional to the measure $\mu_{n}\left(\left\{S_{i_{1}} \cdots S_{i_{n}}(0)\right\}\right)=p_{i_{1}} \cdots p_{i_{n}}$ of the singleton $\left\{S_{i_{1}} \cdots S_{i_{n}}(0)\right\}$.

spectrum of $\mu$ similar to Theorem A. However, we first consider a somewhat more general setting, namely, that of normed structures.

Definition. A normed structure is a pair $(X, N)$ where $X$ is a set and $N: X \rightarrow \mathbb{R}_{+}$is a function such that for all $r>0$, we have

$$
|\{x \in X \mid N(x) \leq r\}|<\infty .
$$

We now list a few examples of normed structures.

ExAmple. Fix $p \geq 1$. Let $X=\mathbb{Z}^{d}$ and put $N(x)=\left(\sum_{i}\left|x_{i}\right|^{p}\right)^{1 / p}$ for $x=\left(x_{1}, \ldots, x_{d}\right) \in \mathbb{Z}^{d}$. Then $(X, N)$ is a normed structure. 
Example. Fix $p \geq 1$. Let $X$ be a lattice in $\mathbb{R}^{d}$ and put $N(x)=$ $\left(\sum_{i}\left|x_{i}\right|^{p}\right)^{1 / p}$ for $x=\left(x_{1}, \ldots, x_{d}\right) \in X$. Then $(X, N)$ is a normed structure.

EXAMPLE. Let $k$ be a number field. Let $\mathfrak{O}$ denote the ring of integers in $k$ and let $\mathcal{J}$ be a subfamily of ideals in $\mathfrak{O}$. For $\mathfrak{x} \in \mathcal{J}$, let $N(\mathfrak{x})$ denote the norm of $\mathfrak{x}$. Then $(\mathcal{J}, N)$ is a normed structure. This example is investigated in detail in Section 2.

EXAMPLE. Let $l$ be a positive integer and let $\mathbb{F}$ be a finite field. For $p \in \mathbb{F}\left[X_{1}, \ldots, X_{l}\right]$, let $N(p)=|\mathbb{F}|^{\operatorname{deg}(p)}$ where $\operatorname{deg}(p)$ denotes the total degree of $p$. Then $\left(F\left[X_{1}, \ldots, X_{l}\right], N\right)$ is a normed structure. This example is investigated in Section 3.

EXAMPLE. Let $G$ be an arithmetical semigroup with norm $N$ (cf. [Kn]). Then $(G, N)$ is a normed structure.

Observe that if $(X, N)$ is a normed structure, then $X$ is countable (this is so since $X=\bigcup_{n \in \mathbb{N}}\{x \in X \mid N(x) \leq n\}$ and each set $\{x \in X \mid N(x) \leq n\}$ is finite). Let $(X, N)$ be a normed structure. If $f: X \rightarrow \mathbb{R}$ is a function and $r \in \mathbb{R}$, we will say that $f(x)$ tends to $r$ as $N(x) \rightarrow \infty$, written

$$
f(x) \rightarrow r \quad \text { as } N(x) \rightarrow \infty,
$$

if the following is satisfied: for each $\varepsilon>0$, there exists $M>0$ such that if $x \in X$ and $N(x) \geq M$, then

$$
|f(x)-r| \leq \varepsilon
$$

Definition. A function $S: X \rightarrow X$ is called power-like if there exist $r>0$ and $t \geq 1$ such that

$$
\frac{N(S(x))}{N(x)^{t}} \rightarrow r \quad \text { as } N(x) \rightarrow \infty .
$$

Motivated by definition (1.1) of a self-similar measure in $\mathbb{R}^{d}$, we define a self-similar measure on $X$ as follows.

Definition. Let $S_{i}: X \rightarrow X$ for $i=1, \ldots, m$ be power-like functions and let $\left(p_{1}, \ldots, p_{m}\right)$ be a probability vector. A measure $\mu$ on $X$ is called self-similar with respect to the family $\left(S_{i}\right)_{i}$ if

$$
\mu=\sum_{i} p_{i} \mu \circ S_{i}^{-1}
$$

The reader will observe that this definition is analogous to that of selfsimilar measures in $\mathbb{R}^{d}$, and our main aim is to formulate and prove a result for $\mu$ similar to Theorem A. Next, we define the lower and upper $L^{q}$ multifractal spectra of a measure $\mu$ on a normed structure. However, first observe that any normed structure is countable and therefore often consists 
of isolated points. Hence, typically $\mu(B(x, r))$ equals 0 for all sufficiently small $r>0$, and so typically we have

$$
\begin{aligned}
\liminf _{r \searrow 0} \frac{\log \int \mu(B(x, r))^{q-1} d \mu(x)}{-\log r} & =\limsup _{r \searrow 0} \frac{\log \int \mu(B(x, r))^{q-1} d \mu(x)}{-\log r} \\
& = \begin{cases}\infty & \text { for } q<1, \\
-\infty & \text { for } 1 \leq q .\end{cases}
\end{aligned}
$$

Hence, in general, the $L^{q}$-spectra $\underline{\tau}_{\mathrm{R}}(q)$ and $\bar{\tau}_{\mathrm{R}}(q)$ introduced in (1.2) do not provide "the correct" way of investigating the multifractal structure of $\mu$. We therefore define the lower and upper $L^{q}$-multifractal spectra of a measure $\mu$ on a normed structure as follows. However, first we introduce some notation.

If $x \in X$, we will abuse notation slightly and write $\mu(x)$ instead of $\mu(\{x\})$. For $r>0$, let

$$
B(r)=\{x \in X \mid N(x) \leq r\} .
$$

Next, fix a measure $\mu$ on $X$. For $E \subseteq X, q \in \mathbb{R}$ and $r>0$, we write

$$
I^{q}(E ; r)=\int_{E \cap B(r)} \mu(x)^{q-1} d \mu(x) .
$$

Observe that since $|\{x \in X \mid N(x) \leq r\}|<\infty$ for all $r>0$, it follows that if $\mu(x)>0$ for all $x \in E$, then $I^{q}(E ; r)$ is finite. We now define the lower and upper q-fractal dimension of $E$ by

$$
\begin{aligned}
\underline{\operatorname{dim}}^{q}(E) & =\liminf _{r \rightarrow \infty} \frac{\log I^{q}(E ; r)}{\log r} \\
& =\liminf _{r \rightarrow \infty} \frac{\log \int_{E \cap B(r)} \mu(x)^{q-1} d \mu(x)}{\log r}, \\
\overline{\operatorname{dim}}^{q}(E) & =\limsup _{r \rightarrow \infty} \frac{\log I^{q}(E ; r)}{\log r} \\
& =\limsup _{r \rightarrow \infty} \frac{\log \int_{E \cap B(r)} \mu(x)^{q-1} d \mu(x)}{\log r} .
\end{aligned}
$$

Write $K$ for the support of $\mu$, i.e.

$$
K=\{x \in X \mid \mu(x)>0\} .
$$

The functions

$$
\underline{\tau}: q \rightarrow \underline{\operatorname{dim}}^{q}(K), \quad \bar{\tau}: q \rightarrow \overline{\operatorname{dim}}^{q}(K),
$$

are called the lower and upper $L^{q}$-multifractal spectra of $\mu$, respectively. We can now state our main result providing a formula similar to (1.3) for the lower and upper $L^{q}$-multifractal spectra of a self-similar measure on a normed structure. 
Theorem 1.1. Let $(X, N)$ be a normed structure. Let $S_{i}: X \rightarrow X$ for $i=1, \ldots, m$ be power-like functions, i.e. for each $i$ there exist $r_{i}>0$ and $t_{i} \geq 1$ such that

$$
\frac{N\left(S_{i}(x)\right)}{N(x)^{t_{i}}} \rightarrow r_{i} \quad \text { as } N(x) \rightarrow \infty .
$$

Let $\left(p_{1}, \ldots, p_{m}\right)$ be a probability vector. Let $\mu$ be a measure on $X$ that is self-similar with respect to the family $\left(S_{i}, p_{i}\right)_{i}$ and write $K$ for the support of $\mu$. Assume that the following three conditions are satisfied:

(i) $r_{i}>1$ for all $i$ with $t_{i}=1$;

(ii) $S_{i}$ is injective for all $i$;

(iii) $S_{i}(K) \cap S_{j}(K)=\emptyset$ for all $i \neq j$.

For each $q \in \mathbb{R}$, define the real number $\beta(q)$ by

$$
\sum_{i: t_{i}=1} p_{i}^{q} \frac{1}{r_{i}^{\beta(q)}}=1
$$

If either (a) $0 \leq \beta(q)$, or (b) $\beta(q)<0$ and $t_{i}=1$ for all $i$, then we have

$$
\underline{\tau}(q)=\bar{\tau}(q)=\beta(q) \text {. }
$$

In Sections 2 and 3 we consider several examples of normed structures taken from algebraic number theory. Finally, Theorem 1.1 is proved in Section 4 . To present the proof we derive two renewal type inequalities for the function $r \mapsto I^{q}(K ; r)$ (cf. (4.3) and (4.9)). For each positive $\delta>0$, these inequalities are seen to imply the existence of a constant $c>0$ such that $I^{q}(K ; r) \leq c r^{\beta(q)+\delta}$ and $I^{q}(K ; r) \geq c r^{\beta(q)-\delta}$ for all $r>0$. Theorem 1.1 follows immediately from this. The reader is referred to [Lal, Ol1] for related (but different) arguments.

If $q=0$, then the dimensions $\underline{\operatorname{dim}}^{q}(E)$ and $\overline{\operatorname{dim}}^{q}(E)$ simplify to

$$
\begin{aligned}
& {\underline{\operatorname{dim}^{0}}}^{(E)}=\liminf _{r \rightarrow \infty} \frac{|\{x \in E \mid N(x) \leq r\}|}{\log r}, \\
& \overline{\operatorname{dim}}^{0}(E)=\limsup _{r \rightarrow \infty} \frac{|\{x \in E \mid N(x) \leq r\}|}{\log r} .
\end{aligned}
$$

For the case where $X=\mathbb{Z}$ and $N(x)=|x|$ for $x \in \mathbb{Z}$, the dimensions in (1.12) have been introduced and studied earlier by various authors; see, for example, [BaT1, BaT2, BeF, Ol2, Ol3].

\section{Example: self-similar multifractals of ideals in number fields.}

We now consider the case where the normed structure is the family of ideals of the ring of integers in a number field. Therefore, let $k$ be a number field and let $\mathfrak{O}$ be the ring of integers in $k$. Let $\mathcal{I}$ denote the family of ideals of $\mathfrak{O}$ and let $\mathcal{J} \subseteq \mathcal{I}$. For $\mathfrak{x} \in \mathcal{I}$, let $N(\mathfrak{x})$ denote the norm of $\mathfrak{x}$. It is well known 
that for each $r>0$, we have

$$
|\{\mathfrak{x} \in \mathcal{I} \mid N(\mathfrak{x}) \leq r\}|<\infty .
$$

In particular, this shows that $(\mathcal{J}, N)$ is a normed structure. In this case we see that the lower and upper $L^{q}$-multifractal spectra of a measure $\mu$ on $\mathcal{J}$ are given by

$$
\begin{gathered}
\underline{\tau}(q)=\liminf _{r \rightarrow \infty} \frac{\log \sum_{\mathfrak{x} \in \mathcal{J}: N(\mathfrak{x}) \leq r} \mu(\mathfrak{x})^{q}}{\log r}, \\
\bar{\tau}(q)=\limsup _{r \rightarrow \infty} \frac{\log \sum_{\mathfrak{x} \in \mathcal{J}: N(\mathfrak{x}) \leq r} \mu(\mathfrak{x})^{q}}{\log r} .
\end{gathered}
$$

By applying Theorem 1.1 to this setting we obtain the following result.

TheOREM 2.1. Let $k$ be a number field and let $\mathfrak{O}$ be the ring of integers in $k$. Let $\mathcal{I}$ denote the family of ideals of $\mathfrak{D}$ and let $\mathcal{J} \subseteq \mathcal{I}$. Let $S_{i}: \mathcal{J} \rightarrow \mathcal{J}$ for $i=1, \ldots, m$ be power-like functions, i.e. for each $i$ there exist $r_{i}>0$ and $t_{i} \geq 1$ such that

$$
\frac{N\left(S_{i}(\mathfrak{x})\right)}{N(\mathfrak{x})^{t_{i}}} \rightarrow r_{i} \quad \text { as } N(\mathfrak{x}) \rightarrow \infty
$$

Let $\left(p_{1}, \ldots, p_{m}\right)$ be a probability vector. Let $\mu$ be a measure on $\mathcal{J}$ that is self-similar with respect to the family $\left(S_{i}, p_{i}\right)_{i}$ and write $K$ for the support of $\mu$. Assume that the following three conditions are satisfied:

(i) $r_{i}>1$ for all $i$ with $t_{i}=1$;

(ii) $S_{i}$ is injective for all $i$;

(iii) $S_{i}(K) \cap S_{j}(K)=\emptyset$ for all $i \neq j$.

For each $q \in \mathbb{R}$, define the real number $\beta(q)$ by

$$
\sum_{i: t_{i}=1} p_{i}^{q} \frac{1}{r_{i}^{\beta(q)}}=1
$$

If either (a) $0 \leq \beta(q)$, or (b) $\beta(q)<0$ and $t_{i}=1$ for all $i$, then we have

$$
\underline{\tau}(q)=\bar{\tau}(q)=\beta(q) .
$$

We are certainly not the first to consider the asymptotic behaviour of sums of the form $\sum_{\mathfrak{x} \in \mathcal{J}, N(\mathfrak{x}) \leq r} \mu(\mathfrak{x})^{q}$ for various choices of $\mu$. Indeed, there is a huge body of literature analyzing this problem for different choices of $\mu$; cf. [Nar, Chapter 7] or [PS, Section 6.6] for numerous examples. However, this appears to be the first study of this problem in the setting of general self-similar measures $\mu$ on $\mathcal{I}$.

ExAmple. Fix $\theta \in \mathfrak{O} \backslash\{0\}$ and let $\mathcal{J}$ be the family of principal ideals $\mathfrak{x}$ of the form $\mathfrak{x}=n \theta \mathfrak{O}$ for $n \in \mathbb{N}$, i.e. $\mathcal{J}=\{n \theta \mathfrak{O} \mid n \in \mathbb{N}\}$. Fix a positive integer $M$ with $M \geq 2$ and let $I \subseteq\{0,1, \ldots, M-1\}$. For $i \in I$, define 
$S_{i}: \mathcal{J} \rightarrow \mathcal{J}$ by

$$
S_{i}(n \theta \mathfrak{O})=(M n+i) \theta \mathfrak{O} .
$$

It is easily seen that $S_{i}$ is well defined and injective for all $i$. We now claim that $S_{i}$ is a power-like function for all $i$; in fact, we will prove that

$$
\frac{N\left(S_{i}(\mathfrak{x})\right)}{N(\mathfrak{x})} \rightarrow M^{[k: \mathbb{Q}]} \quad \text { as } N(\mathfrak{x}) \rightarrow \infty
$$

for all $i$. Indeed, for $\mathfrak{x}=n \theta \mathfrak{O}$ with $n \in \mathbb{N}$, we clearly have

$$
\begin{aligned}
N\left(S_{i}(\mathfrak{x})\right) & =N\left(S_{i}(n \theta \mathfrak{O})\right)=N((M n+i) \theta \mathfrak{O})=|N((M n+i) \theta)| \\
& =|N(M n+i)||N(\theta)|=(M n+i)^{[k: \mathbb{Q}]}|N(\theta)|
\end{aligned}
$$

and

$$
N(\mathfrak{x})=N(n \theta \mathfrak{O})=|N(n \theta)|=|N(n)||N(\theta)|=n^{[k: \mathbb{Q}]}|N(\theta)|
$$

(here we have used the fact that if $x \in \mathbb{Q}$, then $N(x)=x^{[k: \mathbb{Q}]}$ ), whence

$$
\begin{aligned}
\frac{N\left(S_{i}(\mathfrak{x})\right)}{N(\mathfrak{x})} & =\frac{(M n+i)^{[k: \mathbb{Q}]}|N(\theta)|}{n^{[k: \mathbb{Q}]}|N(\theta)|}=\left(M+\frac{i}{n}\right)^{[k: \mathbb{Q}]} \\
& =\left(M+\frac{i}{(N(\mathfrak{x}) /|N(\theta)|)^{1 /[k: \mathbb{Q}]}}\right)^{[k: \mathbb{Q}]} .
\end{aligned}
$$

Equation (2.3) follows immediately from this.

Let $\left(p_{i}\right)_{i \in I}$ be a probability vector and define a measure $\mu$ on $\mathcal{J}$ as follows. For $\mathfrak{x} \in \mathcal{J}$, we put

$$
\mu(\mathfrak{x})= \begin{cases}p_{a_{0}} p_{a_{1}} \cdots p_{a_{n}} & \text { if } \mathfrak{x} \in \mathcal{J} \text { has the form } \\ & \mathfrak{x}=\left(a_{0}+a_{1} M+\cdots+a_{n} M^{n}\right) \theta \mathfrak{O} \\ & \text { for } n \in \mathbb{N} \text { and } a_{0}, a_{1}, \ldots, a_{n} \in I \text { with } a_{n} \neq 0, \\ & \text { otherwise. }\end{cases}
$$

The support $K$ of $\mu$ is clearly equal to

$$
K=\left\{\left(a_{0}+a_{1} M+\cdots+a_{n} M^{n}\right) \theta \mathfrak{O} \mid a_{i} \in I, n \in \mathbb{N}\right\} .
$$

It is not difficult to see that

$$
\mu=\sum_{i \in I} p_{i} \mu \circ S_{i}^{-1}
$$

Indeed, to prove this it clearly suffices to show $\mu(\mathfrak{x})=\sum_{i \in I} p_{i} \mu\left(S_{i}^{-1}(\mathfrak{x})\right)$ for all $\mathfrak{x} \in \mathcal{J}$. For this, let $\mathfrak{x} \in \mathcal{J}$. If $\mathfrak{x} \notin K$, then $S_{i}^{-1}(\mathfrak{x})=\emptyset$ for all $i$, whence $\mu(\mathfrak{x})=0$ and $\sum_{i \in I} p_{i} \mu\left(S_{i}^{-1}(\mathfrak{x})\right)=0$. On the other hand, if $\mathfrak{x} \in K$, then $\mathfrak{x}$ has the form $\mathfrak{x}=\left(a_{0}+a_{1} M+\cdots+a_{n} M^{n}\right) \theta \mathfrak{O}$ for $n \in \mathbb{N}$ and $a_{0}, a_{1}, \ldots, a_{n} \in I$. This implies that

$$
S_{i}^{-1}(\mathfrak{x})= \begin{cases}\left(a_{1}+a_{2} M+\cdots+a_{n} M^{n-1}\right) \theta \mathfrak{O} & \text { if } i=a_{0}, \\ \emptyset & \text { if } i \neq a_{0}\end{cases}
$$


whence

$$
\begin{aligned}
\sum_{i \in I} p_{i} \mu\left(S_{i}^{-1}(\mathfrak{x})\right) & =p_{a_{0}} \mu\left(S_{a_{0}}^{-1}(\mathfrak{x})\right)=p_{a_{0}} \mu\left(\left(a_{1}+a_{2} M+\cdots+a_{n} M^{n-1}\right) \theta \mathfrak{O}\right) \\
& =p_{a_{0}} p_{a_{1}} \cdots p_{a_{n}}=\mu\left(\left(a_{0}+a_{1} M+\cdots+a_{n} M^{n}\right) \theta \mathfrak{O}\right)=\mu(\mathfrak{x}) .
\end{aligned}
$$

This proves (2.4). It is also easily seen that $S_{i}(K) \cap S_{j}(K)=\emptyset$ for all $i \neq j$. It therefore follows from Theorem 2.1 that $\underline{\tau}(q)=\bar{\tau}(q)=\beta(q)$, where $\beta(q)$ is the solution to the equation

whence

$$
\sum_{i \in I} p_{i}^{q} \frac{1}{M^{[k: \mathbb{Q}] \beta(q)}}=1,
$$

$$
\underline{\tau}(q)=\bar{\tau}(q)=\frac{1}{[k: \mathbb{Q}]} \frac{\log \sum_{i \in I} p_{i}^{q}}{\log M} .
$$

If $M=3$ and $I=\{0,2\}$, then the measure $\mu$ is given by

$$
\mu(\mathfrak{x})= \begin{cases}p_{a_{0}} p_{a_{1}} \cdots p_{a_{n}} & \text { if } \mathfrak{x} \in \mathcal{J} \text { has the form } \\ & \mathfrak{x}=\left(a_{0}+a_{1} 3+\cdots+a_{n} 3^{n}\right) \theta \mathfrak{O} \text { for } n \in \mathbb{N} \\ & \text { and } a_{0}, a_{1}, \ldots, a_{n}=0,2 \text { with } a_{n} \neq 0, \\ & \text { otherwise, }\end{cases}
$$

and the set $K$ equals

$$
K=\left\{\left(a_{0}+a_{1} 3+\cdots+a_{n} 3^{n}\right) \theta \mathfrak{O} \mid a_{i}=0,2, n \in \mathbb{N}\right\} .
$$

Figure 2 provides a graphical illustration of the measure $\mu$ in (2.6) for $\left(p_{0}, p_{2}\right)=\left(\frac{2}{3}, \frac{1}{3}\right)$. The reader should note the similarity between this figure and the Cantor measure in Figure 1. It also follows from (2.5) that the lower and upper $L^{q}$-multifractal spectra $\underline{\tau}(q)$ and $\bar{\tau}(q)$ of $\mu$ are given by

$$
\underline{\tau}(q)=\bar{\tau}(q)=\frac{1}{[k: \mathbb{Q}]} \frac{\log \left(p_{0}^{q}+p_{2}^{q}\right)}{\log 3} .
$$

In this case the set $K$ and the measure $\mu$ are clearly discrete ideal analogues of the classical ternary Cantor set $C$ in (1.4) and the Cantor measure in (1.5), respectively, and formula (2.8) for the lower and upper $L^{q}$-multifractal spectra of $\mu$ is clearly analogous to formula (1.6) for the lower and upper $L^{q_{-}}$ multifractal spectra of the Cantor measure.

3. Example: self-similar multifractals in polynomial rings. Let $l$ be a positive integer and let $\mathbb{F}$ be a finite field. We now consider the case where the normed structure is the ring $\mathbb{F}\left[X_{1}, \ldots, X_{l}\right]$ of polynomials of $l$ variables with coefficients in $\mathbb{F}$. For $p \in \mathbb{F}\left[X_{1}, \ldots, X_{l}\right]$, let $\operatorname{deg}(p)$ denote the total degree of $p$, and put $N(p)=|\mathbb{F}|^{\operatorname{deg}(p)}$. It is clear that for each $r>0$, we have $\left|\left\{p \in \mathbb{F}\left[X_{1}, \ldots, X_{l}\right] \mid N(p) \leq r\right\}\right|<\infty$. In particular, this shows that $\left(\mathbb{F}\left[X_{1}, \ldots, X_{l}\right], N\right)$ is a normed structure. In this case we see that the 

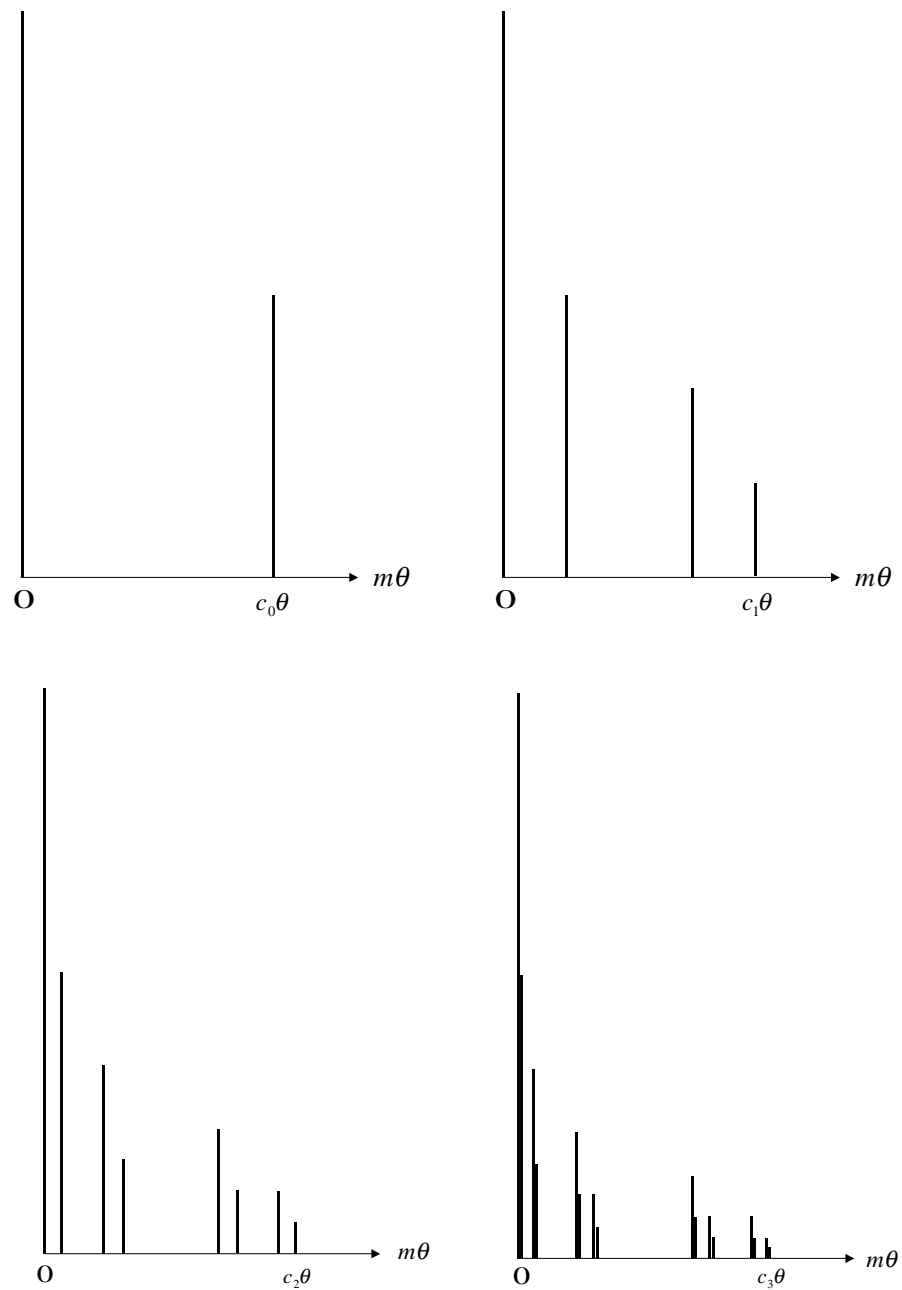

Fig. 2. This figure provides a graphical illustration of the measure $\mu$ in $(2.6)$ for $\left(p_{0}, p_{2}\right)$ $=\left(\frac{2}{3}, \frac{1}{3}\right)$. For $n=0,1,2,3$, the measure restricted to the set $\left\{m \theta \mathfrak{O} \mid m=0,1, \ldots, c_{n}\right\}$, where $c_{n}=2+2 \cdot 3+2 \cdot 3^{2}+\cdots+2 \cdot 3^{n}=3^{n+1}-1$, is sketched. In the figure each principal ideal of the form $m \theta \mathfrak{O}$ with $m \in \mathbb{N}$ is identified with the number $m \theta$, and the height of each vertical bar is proportional to the measure $\mu(m \theta \mathfrak{O})$ of the singleton $\{m \theta \mathfrak{O}\}$.

lower and upper $L^{q}$-multifractal spectra of a measure $\mu$ on $\mathbb{F}\left[X_{1}, \ldots, X_{l}\right]$ are given by

$$
\begin{aligned}
& \underline{\tau}(q)=\liminf _{r \rightarrow \infty} \frac{\log \sum_{p \in \mathbb{F}\left[X_{1}, \ldots, X_{l}\right]:|\mathbb{F}|^{\operatorname{deg}(p)} \leq r} \mu(p)^{q}}{\log r}, \\
& \bar{\tau}(q)=\limsup _{r \rightarrow \infty} \frac{\log \sum_{p \in \mathbb{F}\left[X_{1}, \ldots, X_{l}\right]:|\mathbb{F}|^{\operatorname{deg}(p)} \leq r} \mu(p)^{q}}{\log r} .
\end{aligned}
$$

By applying Theorem 1.1 to this setting we obtain the following result. 
THEOREM 3.1. Let $l$ be a positive integer and let $\mathbb{F}$ be a finite field. Let $S_{i}: \mathbb{F}\left[X_{1}, \ldots, X_{l}\right] \rightarrow \mathbb{F}\left[X_{1}, \ldots, X_{l}\right]$ for $i=1, \ldots, m$ be power-like functions, i.e. for each $i$ there exist $r_{i}>0$ and $t_{i} \geq 1$ such that

$$
\frac{N\left(S_{i}(p)\right)}{N(p)^{t_{i}}} \rightarrow r_{i} \quad \text { as } N(p) \rightarrow \infty .
$$

Let $\left(p_{1}, \ldots, p_{m}\right)$ be a probability vector. Let $\mu$ be a measure on $\mathbb{F}\left[X_{1}, \ldots, X_{l}\right]$ that is self-similar with respect to the family $\left(S_{i}, p_{i}\right)_{i}$ and write $K$ for the support of $\mu$. Assume that the following three conditions are satisfied:

(i) $r_{i}>1$ for all $i$ with $t_{i}=1$;

(ii) $S_{i}$ is injective for all $i$;

(iii) $S_{i}(K) \cap S_{j}(K)=\emptyset$ for all $i \neq j$.

For each $q \in \mathbb{R}$, define the real number $\beta(q)$ by

$$
\sum_{i: t_{i}=1} p_{i}^{q} \frac{1}{r_{i}^{\beta(q)}}=1
$$

If either (a) $0 \leq \beta(q)$, or (b) $\beta(q)<0$ and $t_{i}=1$ for all $i$, then we have

$$
\underline{\tau}(q)=\bar{\tau}(q)=\beta(q) .
$$

Example. For $i=1, \ldots, l$, let $J_{i}$ be a finite set, and let $\left\{a_{i, j} \mid j \in J_{i}\right\}$ be a subset of $\mathbb{F}$. For $i=1, \ldots, l$ and $j$ define $S_{i, j}: \mathbb{F}\left[X_{1}, \ldots, X_{l}\right] \rightarrow$ $\mathbb{F}\left[X_{1}, \ldots, X_{l}\right]$ by

$$
S_{i, j}(p)=X_{i} p+a_{i, j} .
$$

It is easily seen that $S_{i, j}$ is injective, and that

$$
\frac{N\left(S_{i, j}(p)\right)}{N(p)} \rightarrow|\mathbb{F}| \quad \text { as } N(p) \rightarrow \infty
$$

Let $\left(p_{i, j}\right)_{i, j}$ be a probability vector and define a measure $\mu$ on $\mathbb{F}\left[X_{1}, \ldots, X_{l}\right]$ as follows:

$$
\mu(p)=\left\{\begin{array}{l}
p_{i_{0}, j_{0}} p_{i_{1}, j_{1}} \cdots p_{i_{n-1}, j_{n-1}} \\
0
\end{array}\right.
$$$$
\text { if } p \in \mathbb{F}\left[X_{1}, \ldots, X_{l}\right] \text { has the from }
$$$$
p=a_{i_{0}, j_{0}}+a_{i_{1}, j_{1}} X_{i_{0}}+a_{i_{2}, j_{2}} X_{i_{0}} X_{i_{1}}+\cdots
$$$$
\cdots+a_{i_{n}, j_{n}} X_{i_{0}} X_{i_{1}} \cdots X_{i_{n-1}}
$$$$
\text { for } n \in \mathbb{N} \text { with } a_{i_{n}, j_{n}} \neq 0 \text {, }
$$$$
\text { otherwise. }
$$

The support $K$ of $\mu$ is clearly equal to

$$
K=\left\{a_{i_{0}, j_{0}}+a_{i_{1}, j_{1}} X_{i_{0}}+a_{i_{2}, j_{2}} X_{i_{0}} X_{i_{1}}+\cdots+a_{i_{n}, j_{n}} X_{i_{0}} X_{i_{1}} \cdots X_{i_{n-1}} \mid n \in \mathbb{N}\right\} .
$$

It is not difficult to see that

$$
\mu=\sum_{i, j} p_{i, j} \mu \circ S_{i, j}^{-1} .
$$


It is also easily seen that $S_{i_{1}, j_{1}}(K) \cap S_{i_{2}, j_{2}}(K)=\emptyset$ for all $\left(i_{1}, j_{1}\right) \neq\left(i_{2}, j_{2}\right)$. It therefore follows from Theorem 3.1 that $\underline{\tau}(q)=\bar{\tau}(q)=\beta(q)$, where $\beta(q)$ is the solution to the equation

$$
\sum_{i, j} p_{i, j}^{q} \frac{1}{|\mathbb{F}|^{\beta(q)}}=1,
$$

whence

$$
\underline{\tau}(q)=\bar{\tau}(q)=\frac{\log \sum_{i, j} p_{i, j}^{q}}{\log |\mathbb{F}|} .
$$

\section{Proof of Theorem 1.1}

Lemma 4.1. Let $(X, N)$ be a normed structure. Let $S_{i}: X \rightarrow X$ for $i=1, \ldots, m$ be power-like functions. Let $\left(p_{1}, \ldots, p_{m}\right)$ be a probability vector. Let $\mu$ be a measure on $X$ that is self-similar with respect to the family $\left(S_{i}, p_{i}\right)_{i}$ and write $K$ for the support of $\mu$.

(i) If $S_{i}$ is injective for all $i$, then

$$
K=\bigcup_{i} S_{i}(K)
$$

(ii) If $S_{i}$ is injective for all $i$ and $S_{i}(K) \cap S_{j}(K)=\emptyset$ for all $i \neq j$, then

$$
\mu\left(S_{i} x\right)=p_{i} \mu(x)
$$

for all $x \in K$.

Proof. (i) We first prove that $K \subseteq \bigcup_{i} S_{i}(K)$. Let $x \in K$. It follows from (1.7) that $0<\mu(x)=\sum_{i} p_{i} \mu\left(S_{i}^{-1} x\right)$, and we therefore conclude that there exists $j$ such that $\mu\left(S_{j}^{-1} x\right)>0$. This shows that $S_{j}^{-1} x \subseteq K$, whence $x \in$ $S_{j}(K) \subseteq \bigcup_{i} S_{i}(K)$. Next, we prove that $\bigcup_{i} S_{i}(K) \subseteq K$. Let $x \in \bigcup_{i} S_{i}(K)$. In particular, this implies that there exists $j$ such that $x \in S_{j}(K)$, whence $S_{j}^{-1} x \in K$, and so $\mu\left(S_{j}^{-1} x\right)>0$. Again, using (1.7), we infer that $\mu(x)=$ $\sum_{i} p_{i} \mu\left(S_{i}^{-1} x\right) \geq p_{j} \mu\left(S_{j}^{-1} x\right)>0$. This shows that $x \in K$.

(ii) Using (1.7) we see that

$$
\mu\left(S_{i} x\right)=\sum_{j} p_{j} \mu\left(S_{j}^{-1} S_{i} x\right) .
$$

However, since $S_{l}$ is injective for all $l$ and $S_{l}(K) \cap S_{j}(K)=\emptyset$ for all $l \neq j$, we conclude that $S_{j}^{-1} S_{i} x=\emptyset$ for $i \neq j$ and that $S_{i}^{-1} S_{i} x=x$. Hence

$$
\mu\left(S_{i} x\right)=\sum_{j} p_{j} \mu\left(S_{j}^{-1} S_{i} x\right)=p_{i} \mu\left(S_{i}^{-1} S_{i} x\right)=p_{i} \mu(x) .
$$

This completes the proof. 
Proof of Theorem 1.1. Part 1. We must prove that $\bar{\tau}(q) \leq \beta(q)$. Fix $\delta>0$. It follows from the definition of $\beta(q)$ that

$$
1=\sum_{i: t_{i}=1} p_{i}^{q} \frac{1}{r_{i}^{\beta(q)}}>\sum_{i: t_{i}=1} p_{i}^{q} \frac{1}{r_{i}^{\beta(q)+\delta}},
$$

and we can thus choose $\varepsilon>0$ such that

$$
\sum_{i: t_{i}=1} p_{i}^{q} \frac{1}{\left(r_{i}-\varepsilon\right)^{\beta(q)+\delta}}<1 .
$$

Recall that either (a) $0 \leq \beta(q)$, or (b) $\beta(q)<0$ and $t_{i}=1$ for all $i$. This combined with the previous inequality implies that there exists $R_{0}>0$ such that

$$
\begin{aligned}
& \sum_{i: t_{i}=1} p_{i}^{q} \frac{1}{\left(r_{i}-\varepsilon\right)^{\beta(q)+\delta}} \\
& \quad+\sum_{i: t_{i} \neq 1} p_{i}^{q} \frac{1}{\left(r_{i}-\varepsilon\right)^{(\beta(q)+\delta) / t_{i}}} \frac{1}{r^{\left(1-1 / t_{i}\right)(\beta(q)+\delta)}}<1
\end{aligned}
$$

for all $r>R_{0}$.

Next, observe that since $N\left(S_{i}(x)\right) / N(x)^{t_{i}} \rightarrow r_{i}$ as $N(x) \rightarrow \infty$, there exists a positive number $M>0$ such that

$$
\left|\frac{N\left(S_{i}(x)\right)}{N(x)^{t_{i}}}-r_{i}\right| \leq \varepsilon
$$

for all $x \in X$ with $N(x) \geq M$ and all $i$.

Finally, write

$$
R=\max \left(R_{0},\left(M \max _{i}\left(r_{i}-\varepsilon\right)^{1 / t_{i}}\right)^{\max _{i} t_{i}}\right) .
$$

Claim 1. For all $r \geq R$ we have

$$
S_{i}(K) \cap B(r) \subseteq S_{i}\left(K \cap B\left(\left(\frac{r}{r_{i}-\varepsilon}\right)^{1 / t_{i}}\right)\right)
$$

for all $i$.

Proof of Claim 1. Let $x \in S_{i}(K) \cap B(r)$. We must now prove that $S_{i}(x) \in$ $S_{i}\left(K \cap B\left(\left(r /\left(r_{i}-\varepsilon\right)\right)^{1 / t_{i}}\right)\right)$. Since $x \in S_{i}(K) \cap B(r)$, there exists $u \in K$ such that $x=S_{i}(u)$. We have to show that $u \in K \cap B\left(\left(r /\left(r_{i}-\varepsilon\right)\right)^{1 / t_{i}}\right)$. It is clear that $u \in K$. Hence we must show that $N(u) \leq\left(r /\left(r_{i}-\varepsilon\right)\right)^{1 / t_{i}}$. There are two cases to consider.

CASE 1: $M \leq N(u)$. In this case it follows from (4.2) that

$$
\left|\frac{N\left(S_{i}(u)\right)}{N(u)^{t_{i}}}-r_{i}\right| \leq \varepsilon
$$


Rearranging this inequality shows that

$$
N(u) \leq\left(\frac{N\left(S_{i}(u)\right)}{r_{i}-\varepsilon}\right)^{1 / t_{i}}=\left(\frac{N(x)}{r_{i}-\varepsilon}\right)^{1 / t_{i}} \leq\left(\frac{r}{r_{i}-\varepsilon}\right)^{1 / t_{i}} .
$$

CASE 2: $N(u) \leq M$. Since $r \geq R \geq\left(M \max _{i}\left(r_{i}-\varepsilon\right)^{1 / t_{i}}\right)^{\max _{i} t_{i}}$, we see that $M \leq\left(r /\left(r_{i}-\varepsilon\right)\right)^{1 / t_{i}}$. Hence, in Case 2 we also have

$$
N(u) \leq M \leq\left(\frac{r}{r_{i}-\varepsilon}\right)^{1 / t_{i}}
$$

This completes the proof of Claim 1.

Since (by Lemma 4.1) $K=\bigcup_{i} S_{i}(K)$, it follows from Claim 1 that for all $r \geq R$ we have

$$
\begin{aligned}
I^{q}(K ; r) & =\sum_{x \in K \cap B(r)} \mu(x)^{q} \leq \sum_{i} \sum_{x \in S_{i}(K) \cap B(r)} \mu(x)^{q} \\
& \leq \sum_{i} \sum_{S_{i}\left(K \cap B\left(\left(\frac{r}{r_{i}-\varepsilon}\right)^{1 / t_{i}}\right)\right)} \mu(x)^{q}=\sum_{i} \sum_{u \in K \cap B\left(\left(\frac{r}{r_{i}-\varepsilon}\right)^{1 / t_{i}}\right)} \mu\left(S_{i} x\right)^{q} .
\end{aligned}
$$

Finally, by Lemma 4.1, this implies that

$$
\begin{aligned}
I^{q}(K ; r) & \leq \sum_{i} \sum_{u \in K \cap B\left(\left(\frac{r}{r_{i}-\varepsilon}\right)^{1 / t_{i}}\right)} p_{i}^{q} \mu(x)^{q} \\
& =\sum_{i} p_{i}^{q} I^{q}\left(\left(\frac{r}{r_{i}-\varepsilon}\right)^{1 / t_{i}}\right) .
\end{aligned}
$$

Next, define $W: \mathbb{R}^{+} \rightarrow \mathbb{R}$ by

$$
W(r)=r^{-(\beta(q)+\delta)} I^{q}(K ; r) .
$$

It follows from (4.1) and (4.3) that if $r \geq R$, then

$$
\begin{aligned}
& W(r) \leq r^{-(\beta(q)+\delta)} \sum_{i} p_{i}^{q}\left(\frac{r}{r_{i}-\varepsilon}\right)^{(\beta(q)+\delta) / t_{i}} W\left(\left(\frac{r}{r_{i}-\varepsilon}\right)^{1 / t_{i}}\right) \\
= & \sum_{i} p_{i}^{q} \frac{1}{\left(r_{i}-\varepsilon\right)^{(\beta(q)+\delta) / t_{i}}} \frac{1}{r^{\left(1-1 / t_{i}\right)(\beta(q)+\delta)}} W\left(\left(\frac{r}{r_{i}-\varepsilon}\right)^{1 / t_{i}}\right) \\
\leq & \left(\sum_{i} p_{i}^{q} \frac{1}{\left(r_{i}-\varepsilon\right)^{(\beta(q)+\delta) / t_{i}}} \frac{1}{r^{\left(1-1 / t_{i}\right)(\beta(q)+\delta)}}\right) \sup _{j} W\left(\left(\frac{r}{r_{j}-\varepsilon}\right)^{1 / t_{j}}\right)
\end{aligned}
$$




$$
\begin{aligned}
& =\left(\sum_{i: t_{i}=1} p_{i}^{q} \frac{1}{\left(r_{i}-\varepsilon\right)^{\beta(q)+\delta}}+\sum_{i: t_{i} \neq 1} p_{i}^{q} \frac{1}{\left(r_{i}-\varepsilon\right)^{(\beta(q)+\delta) / t_{i}}} \frac{1}{r^{\left(1-1 / t_{i}\right)(\beta(q)+\delta)}}\right) \\
& \quad \times \sup _{j} W\left(\left(\frac{r}{r_{j}-\varepsilon}\right)^{1 / t_{j}}\right) \\
& \leq \sup _{j} W\left(\left(\frac{r}{r_{j}-\varepsilon}\right)^{1 / t_{j}}\right) .
\end{aligned}
$$

Now, write $\Delta=\min _{i}\left(r_{i}-\varepsilon\right)^{1 / t_{i}}>1$. Next, we prove that for all positive integers $l$ with $R \leq \Delta^{l-1}$, we have

$$
\sup _{0<r \leq \Delta^{l}} W(r) \leq \sup _{0<r \leq \Delta^{l-1}} W(r) .
$$

Indeed, for $r$ with $R \leq r \leq \Delta^{l}$, we have

$$
\left(\frac{r}{r_{j}-\varepsilon}\right)^{1 / t_{j}}=\frac{r^{1 / t_{j}}}{\left(r_{j}-\varepsilon\right)^{1 / t_{j}}} \leq \frac{r}{\Delta} \leq \frac{\Delta^{l}}{\Delta}=\Delta^{l-1}
$$

for all $j$, and (4.4) therefore implies that

$$
W(r) \leq \sup _{j} W\left(\left(\frac{r}{r_{j}-\varepsilon}\right)^{1 / t_{j}}\right) \leq \sup _{0<\varrho \leq \Delta^{l-1}} W(\varrho) .
$$

This proves (4.5).

Choose a positive integer $l_{0}$ with $\Delta^{l_{0}} \geq R$. Successive applications of (4.5) yield

$$
\begin{aligned}
\sup _{0<r} W(r) & =\sup _{l_{0}<l} \sup _{0<r \leq \Delta^{l}} W(r) \\
& \leq \sup _{l_{0}<l} \sup _{0<r \leq \Delta^{l-1}} W(r) \\
& \vdots \\
& \leq \sup _{l_{0}<l} \sup _{0<r \leq \Delta^{l_{0}}} W(r) \\
& =\sup _{0<r \leq \Delta^{l_{0}}} W(r) .
\end{aligned}
$$

Since clearly $c=\sup _{0<r \leq \Delta^{l_{0}}} W(r)=\sup _{0<r \leq \Delta^{l_{0}}} r^{-(\beta(q)+\delta)} I^{q}(K ; r)<\infty$ (because $I^{q}(K ; r)=0$ for all sufficiently small $r \neq 0$ ), we conclude from (4.6) that $r^{-(\beta(q)+\delta)} I^{q}(K ; r)=W(r) \leq c$ for all $r>0$. This clearly implies

$$
\bar{\tau}(q)=\limsup _{r \rightarrow \infty} \frac{\log I^{q}(K ; r)}{\log r} \leq \beta(q)+\delta .
$$

Letting $\delta \searrow 0$ gives the desired result. 
Proof of Theorem 1.1. Part 2. We must prove that $\beta(q) \leq \underline{\tau}(q)$. Fix $\delta>0$. It follows from the definition of $\beta(q)$ that

$$
1=\sum_{i: t_{i}=1} \frac{1}{r_{i}^{\beta(q)}}<\sum_{i: t_{i}=1} \frac{1}{r_{i}^{\beta(q)-\delta}},
$$

and we can thus choose $\varepsilon>0$ such that

$$
\sum_{i: t_{i}=1} \frac{1}{\left(r_{i}+\varepsilon\right)^{\beta(q)-\delta}}>1 \text {. }
$$

Next, observe that since $N\left(S_{i}(x)\right) / N(x)^{t_{i}} \rightarrow r_{i}$ as $N(x) \rightarrow \infty$, there exists a positive number $M>0$ such that

$$
\left|\frac{N\left(S_{i}(x)\right)}{N(x)}-r_{i}\right| \leq \varepsilon
$$

for all $x \in X$ with $N(x) \geq M$ and all $i$ with $t_{i}=1$.

Finally write

$$
R=\sup _{i: N(x) \leq M} N\left(S_{i}(x)\right)<\infty .
$$

Claim 2. For all $r \geq R$ we have

$$
S_{i}\left(K \cap B\left(\frac{r}{r_{i}+\varepsilon}\right)\right) \subseteq S_{i}(K) \cap B(r)
$$

for all $i$ with $t_{i}=1$.

Proof of Claim 2. Let $x \in K \cap B\left(\frac{r}{r_{i}+\varepsilon}\right)$. We must now prove that $S_{i}(x) \in$

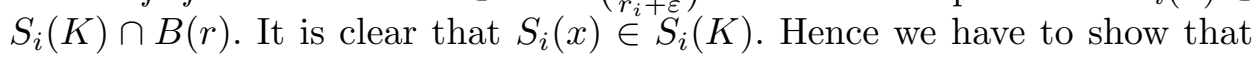
$\left|S_{i}(x)\right| \leq r$.

CASE 1: $M \leq N(x)$. In this case it follows from (4.8) that

$$
\left|\frac{N\left(S_{i}(x)\right)}{N(x)}-r_{i}\right| \leq \varepsilon \text {. }
$$

Also, $x \in K \cap B\left(\frac{r}{r_{i}+\varepsilon}\right)$, whence $N(x) \leq \frac{r}{r_{i}+\varepsilon}$. Rearranging this inequality shows that $\left(r_{i}+\varepsilon\right) N(x) \leq r$. Hence

$$
\begin{aligned}
N\left(S_{i}(x)\right) & =r_{i} N(x)+N(x)\left(\frac{N\left(S_{i}(x)\right)}{N(x)}-r_{i}\right) \\
& \leq r_{i} N(x)+N(x)\left|\frac{N\left(S_{i}(x)\right)}{N(x)}-r_{i}\right| \\
& \leq r_{i} N(x)+N(x) \varepsilon \leq r .
\end{aligned}
$$

Case 2: $N(x) \leq M$. Since $N(x) \leq M$ and $r \geq R$, we see that

$$
N(x) \leq \sup _{i: N(y) \leq M} N\left(S_{i}(y)\right)=R \leq r .
$$

This completes the proof of Claim 2 . 
Since $K=\bigcup_{i} S_{i}(K)$ and $S_{i}(K) \cap S_{j}(K)=\emptyset$ for $i \neq j$, it follows from Claim 2 that for all $r \geq R$ we have

$$
\begin{aligned}
I^{q}(K ; r) & =\sum_{x \in K \cap B(r)} \mu(x)^{q}=\sum_{i} \sum_{x \in S_{i}(K) \cap B(r)} \mu(x)^{q} \\
& \geq \sum_{i: t_{i}=1} \sum_{x \in S_{i}(K) \cap B(r)} \mu(x)^{q} \geq \sum_{i: t_{i}=1} \sum_{x \in S_{i}\left(K \cap B\left(\frac{r}{r_{i}+\varepsilon}\right)\right)} \mu(x)^{q} \\
& \geq \sum_{i: t_{i}=1} \sum_{u \in K \cap B\left(\frac{r}{r_{i}+\varepsilon}\right)} \mu\left(S_{i} u\right)^{q} .
\end{aligned}
$$

Finally, by Lemma 4.1, this implies that

$$
I^{q}(K ; r) \geq \sum_{i: t_{i}=1} \sum_{u \in K \cap B\left(\frac{r}{r_{i}+\varepsilon}\right)} p_{i}^{q} \mu(u)^{q}=\sum_{i: t_{i}=1} p_{i}^{q} I^{q}\left(\frac{r}{r_{i}+\varepsilon}\right) .
$$

Next, define $W: \mathbb{R}^{+} \rightarrow \mathbb{R}$ by

$$
W(r)=r^{-(\beta(q)-\delta)} I^{q}(K ; r) .
$$

It follows from (4.7) and (4.9) that if $r \geq R$, then

$$
\begin{aligned}
W(r) & \geq r^{-(\beta(q)-\delta)} \sum_{i: t_{i}=1} p_{i}^{q}\left(\frac{r}{r_{i}+\varepsilon}\right)^{\beta(q)-\delta} W\left(\frac{r}{r_{i}+\varepsilon}\right) \\
& =\sum_{i: t_{i}=1} p_{i}^{q} \frac{1}{\left(r_{i}+\varepsilon\right)^{\beta(q)-\delta}} W\left(\frac{r}{r_{i}+\varepsilon}\right) \\
& \geq\left(\sum_{i: t_{i}=1} p_{i}^{q} \frac{1}{\left(r_{i}+\varepsilon\right)^{\beta(q)-\delta}}\right) \inf _{j: t_{j}=1} W\left(\frac{r}{r_{j}+\varepsilon}\right) \\
& \geq \inf _{j: t_{j}=1} W\left(\frac{r}{r_{j}+\varepsilon}\right) .
\end{aligned}
$$

Now, write $\Delta=\min _{i}\left(r_{i}+\varepsilon\right)>1$. Next, we prove that for all positive integers $l$ with $R \leq \Delta^{l-1}$, we have

$$
\inf _{0<r \leq \Delta^{l}} W(r) \geq \inf _{0<r \leq \Delta^{l-1}} W(r) .
$$

Indeed, for $r$ with $R \leq r \leq \Delta^{l}$, we have

$$
\frac{r}{r_{j}+\varepsilon} \leq \frac{r}{\Delta} \leq \frac{\Delta^{l}}{\Delta}=\Delta^{l-1}
$$

for all $j$, and (4.10) therefore implies that

$$
W(r) \geq \inf _{j: t_{j}=1} W\left(\frac{r}{r_{j}+\varepsilon}\right) \geq \inf _{0<\varrho \leq \Delta^{l-1}} W(\varrho) .
$$

This proves (4.11). 
Choose a positive integer $l_{0}$ with $\Delta^{l_{0}} \geq R$. Successive applications of (4.11) yields

$$
\begin{aligned}
\inf _{0<r} W(r) & =\inf _{l_{0}<l} \inf _{0<r \leq \Delta^{l}} W(r) \\
& \geq \inf _{l_{0}<l} \inf _{0<r \leq \Delta^{l-1}} W(r) \\
& \vdots \\
& \geq \inf _{l_{0}<l} \inf _{0<r \leq \Delta^{l_{0}}} W(r) \\
& =\inf _{0<r \leq \Delta^{l_{0}}} W(r) .
\end{aligned}
$$

Since clearly $c=\inf _{0<r \leq \Delta^{l_{0}}} W(r)=\inf _{0<r \leq \Delta^{l_{0}}} r^{-(\beta(q)-\delta)} I^{q}(K ; r)>0$, we conclude from (4.12) that $r^{-(\beta(q)-\delta)} I^{q}(K ; r)=W(r) \geq c$ for all $r>0$. This clearly implies

$$
\underline{\tau}(q)=\liminf _{r \rightarrow \infty} \frac{\log I^{q}(K ; r)}{\log r} \geq \beta(q)-\delta .
$$

Letting $\delta \searrow 0$ gives the desired result.

\section{References}

[AP] M. Arbeiter and N. Patzschke, Random self-similar multifractals, Math. Nachr. 181 (1996), 5-42.

[BaT1] M. T. Barlow and S. J. Taylor, Fractional dimension of sets in discrete spaces, with a reply by J. Naudts, J. Phys. A 22 (1989), 2621-2628.

[BaT2] -, - Defining fractal subsets of $\mathbb{Z}^{d}$, Proc. London Math. Soc. 64 (1992), $125-152$.

[BeF] T. Bedford and A. Fisher, Analogues of the Lebesgue density theorem for fractal sets of reals and integers, ibid., 95-124.

[Ed] G. Edgar, Integral, Probability, and Fractal Measures, Springer, New York, 1998.

[Fa] K. J. Falconer, Techniques in Fractal Geometry, Wiley, 1997.

[HJKPS] T. C. Halsey, M. H. Jensen, L. P. Kadanoff, I. Procaccia and B. J. Shraiman, Fractal measures and their singularities: The characterization of strange sets, Phys. Rev. A 33 (1986), 1141-1151.

[Hu] J. Hutchinson, Fractals and self-similarity, Indiana Univ. Math. J. 30 (1981), 713-747.

[Kn] J. Knopfmacher, Abstract Analytic Number Theory, North-Holland Math. Library 12, North-Holland, Amsterdam, 1975.

[Lal] S. Lalley, The packing and covering functions of some self-similar fractals, Indiana Univ. Math. J. 37 (1988), 699-710.

[Lau K. S. Lau, Self-similarity, $L^{p}$-spectrum and multifractal formalism, in: Fractal Geometry and Stochastics (Finsterbergen, 1994), Progr. Probab. 37, Birkhäuser, Basel, 1995, 55-90. 
[Nar] W. Narkiewicz, Elementary and Analytic Theory of Algebraic Numbers, 2nd ed., PWN, Warszawa, and Springer, Berlin, 1990.

[Ol1] L. Olsen, Dimensions of overlaps of self-similar fractals and self-similar multifractals, Indiana Univ. Math. J. 51 (2002), 1461-1477.

[Ol2] - Distribution of digits in integers: fractal dimensions and zeta functions, Acta Arith. 105 (2002), 253-277.

[O13] -, Distribution of digits in integers: Besicovitch-Eggleston subsets of $\mathbb{N}$, J. London Math. Soc. 67 (2003), 561-579.

[PS] A. N. Parshin and I. R. Shafarevich (eds.), Number Theory. II. Algebraic Number Theory, Encyclopaedia Math. Sci. 62, Springer, Berlin, 1992; transl. of: V. G. Chirskiı̌ (ed.), Number Theory. 2, Itogi Nauki i Tekhniki 62; Vsesoyuz. Inst. Nauchn. i Tekhn. Inform., Akad. Nauk SSSR, Moscow, 1990.

[Ra] D. Rand, The singularity spectrum $f(\alpha)$ for cookie-cutters, Ergodic Theory Dynam. Systems 9 (1989), 527-541.

[St] R. Strichartz, Self-similar measures and their Fourier transforms. III, Indiana Univ. Math. J. 42 (1993), 367-411.

Department of Mathematics

University of St. Andrews

St. Andrews, Fife KY16 9SS, Scotland

E-mail: lo@st-and.ac.uk 